\title{
A Case of Streptococcus gallolyticus subsp. gallolyticus Infective Endocarditis with Colon Cancer: Identification by $16 S$ Ribosomal DNA Sequencing
}

\author{
Seon Young Kim, M.D., Sei-Ick Joo, M.T., Jongyoun Yi, M.D., and Eui-Chong Kim, M.D. \\ Department of Laboratory Medicine, Seoul National University Hospital, Seoul, Korea
}

\begin{abstract}
Although the association between Streptococcus bovis endocarditis and colon carcinoma is well known, very few cases of $S$. bovis infection associated with underlying malignancies have been reported in Korea. The S. bovis group has been recently reclassified and renamed as Streptococcus gallolyticus and Streptococcus infantarius subspecies under a new nomenclature system. We report a case of infective endocarditis with colon cancer caused by $S$. gallolyticus subsp. gallolyticus (previously named S. bovis biotype I). A 59-yr-old woman presented with a 1-month history of fever. Initial blood cultures were positive for gram-positive cocci, and echocardiography showed vegetation on mitral and aortic valves. Antibiotic treatment for infective endocarditis was started. The infecting strain was a catalase-negative and bile-esculin-positive alpha-hemolytic Streptococcus susceptible to penicillin and vancomycin. The strain was identified as $S$. gallolyticus subsp. gallolyticus with the use of the Vitek 2 GPI and API 20 Strep systems (bioMérieux, USA). The 16S rDNA sequences of the blood culture isolates showed $100 \%$ homology with those of S. gallolyticus subsp. gallolyticus reported in GenBank. The identification of the infecting organism, and the subsequent communication among clinical microbiologists and physicians about the changed nomenclature, led to the detection of colon cancer. The patient recovered after treatment with antibiotics, valve surgery, and operation for colon cancer. This is the first report of biochemical and genetic identification of $S$. gallolyticus subsp. gallolyticus causing infective endocarditis associated with underlying colon cancer in a Korean patient. (Korean J Lab Med 2010;30:160-5)
\end{abstract}

Key Words : Streptococcus gallolyticus, Endocarditis, Colon cancer, rDNA sequencing

\section{INTRODUCTION}

Streptococcus bovis is a member of group D streptococcicommon inhabitants of the intestine and causative agents of endocarditis. A well-recognized association has been established between $S$. bovis endocarditis and colon carcinoma, which led to great interest in the identification of

Received : November 23, 2009

Manuscript No : KJLM09-135

Revision received: January 14, 2010

Accepted : February 19, 2010

Corresponding author : Eui-Chong Kim, M.D.

Department of Laboratory Medicine, Seoul National University

College of Medicine, 28 Yeongeon-dong, Jongno-gu,

Seoul 110-744, Korea

Tel : +82-2-2072-3500 Fax : +82-2-764-6542

E-mail : euichong@snu.ac.kr this organism [1]. The $S$. bovis group is divided into 3 biotypes according to their biochemical characteristics: biotype I (mannitol fermentation positive), biotype II/1 (mannitol negative and $\beta$-glucuronidase negative), and biotype II/2 (mannitol negative and $\beta$-glucuronidase positive) [2]. Each biotype has somewhat different pathogenicity. $S$. bovis biotype I has been documented to be the biotype more likely associated with both endocarditis and malignant or premalignant colonic lesions [3]. The taxonomy of the $S$. bovis group has been evolving in the last few decades, and a new nomenclature was adopted on the basis of genetic distances and phylogenetic analyses. The new classification lists $S$. bovis biotypes I, II/1, and II/2 as Streptococcus gallolyticus subsp. gallolyticus, Streptococcus infantarius 
subsp. coli, and S. gallolyticus subsp. pasteurianus, respectively [4]. We report a case of infective endocarditis with colon cancer caused by S. gallolyticus subsp. gallolyticus. Identification of the causative organism was performed by phenotypic characterization and 16S rDNA sequence analysis.

\section{CASE REPORT}

A 59-yr-old woman was admitted to Seoul National University Hospital, with a 1-month history of recurrent episodes of fever and chills. She also had arthralgia of both knees and 3 episodes of fecal incontinence for 1 month. She was a carrier of the hepatitis $\mathrm{C}$ virus and had a history of stage 4 gastric marginal zone lymphoma, which had been previously treated with chemotherapy over 2 yr ago. She had no evidence of residual lymphoma after treatment. On physical examination, fever was present $\left(38.9^{\circ} \mathrm{C}\right)$; lung fields were clear; heart sounds were regular without murmur; and abdomen was soft, non-distended, and with no palpable mass. Digital rectal examination revealed no melena, hematochezia, or mass. Specimens for laboratory tests and blood cultures were collected, and empirical antibiotic therapy with intravenous ciprofloxacin (400 mg every 12 hr) was started. Initial laboratory studies showed the following results: hemoglobin (11.1 g/dL), white blood cells [12.01 $\times 10^{9} / \mathrm{L}$; neutrophils (79\%), lymphocytes (14\%), and monocytes (4\%)], platelets $\left(242 \times 10^{9} / \mathrm{L}\right)$, and $\mathrm{C}$-reactive protein $(5.79 \mathrm{mg} / \mathrm{dL})$. Results of the liver function tests and creatinine, glucose, and troponin tests were within the normal range. Stool occult blood test was not performed. The patient's chest radiograph was unremarkable. She had persistent fever increasing up to $39.1^{\circ} \mathrm{C}$ despite antibiotic therapy, and initial blood cultures were positive for grampositive cocci in pairs and short chains. Twenty-four hours later, ciprofloxacin was discontinued and vancomycin (1,000 mg every $12 \mathrm{hr}$ ) was started after collecting additional blood cultures. An echocardiography was performed, which showed vegetations on the mitral and aortic valves. The initial isolate from the first blood cultures was identified as $S$. gallolyticus subsp. gallolyticus, which was susceptible to penicillin. The second blood cultures were also positive for similar alpha-hemolytic streptococci. Vancomycin was discontinued and antibiotics were replaced by intravenous penicillin G (3,000,000 U every $4 \mathrm{hr}$ ) and gentamicin (63 mg every $8 \mathrm{hr}$ ). The fever subsided, and the subsequent blood cultures were negative for streptococci. The isolate from the second blood cultures was initially identified as Streptococcus mutans; however, the results of retest and further investigation confirmed that it was the same strain as the isolate from the first blood cultures. The results of blood cultures were discussed among clinical microbiologists and physicians, including the corrected results of the second blood cultures, the information about the causative organism (i.e., S. gallolyticus subsp. gallolyticus, which was named as $S$. bovis biotype I in the previous nomenclature system), and the risk of colon cancer. The abdomen computerized tomography (CT) image showed newly detected segmental wall thickening of the sigmoid colon. Colonoscopy revealed an ulceroinfiltrative mass, which was confirmed as adenocarcinoma. The patient underwent operation for valve replacement and anterior resection for colon cancer on the 18th and 50th day of hospitalization, respectively. She successfully recovered and was discharged after completing the antibiotic therapy and postoperative care.

Both the first and the second blood cultures showed gram-positive cocci in pairs and short chains after $24 \mathrm{hr}$ of incubation in broths at $37^{\circ} \mathrm{C}$ (Bactec, Becton Dickinson, Baltimore, MD, USA). Subsequent subculture on 5\% sheep blood and chocolate agars incubated at $35^{\circ} \mathrm{C}$ showed small alpha-hemolytic colonies. The isolates were negative for catalase and pyrrolidonylarylaminidase, showed no zone of inhibition around the optochin disk, failed to grow in heart infusion broth containing $6.5 \% \mathrm{NaCl}$, and bile-esculin positive after overnight incubation. A tentative identification of viridans group Streptococcus was made. The Vitek2 GP identification system (bioMérieux, Durham, NC, USA) was used to identify the isolate from the first blood culture as S. gallolyticus subsp. gallolyticus; the isolate from the second culture was identified as $S$. mutans. The tests were repeated twice for the subculture of the second isolate, and the results were as follows: S. gallolyticus subsp. gallolyti- 
cus (excellent identification, probability 98.11\%), S. gallolyticus subsp. gallolyticus (low discrimination, 50.52\%), and S. mutans (49.48\%). Both of the repeated tests showed identical biochemical profiles, except that the test with an excellent confidence level showed a positive result in the pullulan acidification test. The API 20 Strep system (bioMérieux, Durham, NC, USA) identified the isolate from the first blood culture as $S$. bovis biotype I (S. gallolyticus subsp. gallolyticus) (profile 5000453, 78.1\%) and S. bovis biotype II (13.5\%), while the isolate from the second culture was identified as $S$. bovis biotype I (S. gallolyticus subsp. gallolyticus) (profile 5000553, 99.1\%). The first isolate showed negative reactions with leucine arylamidase and mannitol, which are atypical for $S$. gallolyticus subsp. gallolyticus. The subculture of the first isolate was tested repeatedly and identified by the API 20 Strep system as $S$. gallolyticus subsp. gallolyticus, with $99.1 \%$ probability. The biochemical profiles of this strain are compared with those of the S. gallolyticus and $S$. infantarius subspecies in Table 1. Antimicrobial susceptibility testing was performed by $\mathrm{E}$ test for penicillin and disk diffusion methods for other antibiotics. Isolates from the first and second blood cultures showed identical results: susceptible to penicillin (MIC= $0.032 \mu \mathrm{g} / \mathrm{mL}$ ), vancomycin, and chloramphenicol and resistant to erythromycin and clindamycin.

We performed 16S rDNA gene sequencing for the isolates from the first and second blood cultures. The forward (27F: 5'-AGAGTTTGATCMTGGCTCAG-3') and reverse (1492R: 5'-TACGGYTACCTTGTTACGACTT-3') primers were used to amplify $16 \mathrm{~S}$ rDNA gene fragments, representing approximately $98 \%$ of the complete $16 \mathrm{~S}$ rDNA gene. The following primers were used for sequencing of the fragments obtained: 27F, 518F (5'-CCAGCAGCCGCGGTAATACG-3') and 800R (5'-TACCAGGGTATCTAATCC-3'). Both isolates showed identical 16S rDNA gene sequences and showed 100\% (1,480/1,480 bp) homology with those of $S$. gallolyticus subsp. gallolyticus (EU163500, GenBank). Isolates from the first and second blood cultures showed 99.7\% (1,476/ 1,480 bp) and 98.6\% (1,459/1,480 bp) homology with $S$. gallolyticus subsp. pasteurianus (EU163502, GenBank) and $S$. infantarius subsp. coli (EU163503, GenBank), respectively [5]. The sequences were analyzed by using MicroSeq ID 16S rDNA 500 library v2.1 and analysis software v2.0 (Applied Biosystems, Foster City, CA, USA), and the isolate was

Table 1. Characteristics of the blood culture isolates of this case in comparison with the subspecies of Streptococcus gallolyticus and Streptococcus infantarius

\begin{tabular}{|c|c|c|c|c|c|c|c|}
\hline \multirow[b]{2}{*}{ Characteristics } & \multicolumn{2}{|c|}{ Isolates of this case } & \multicolumn{5}{|c|}{ S. gallolyticus and S. infantarius subspecies* } \\
\hline & $\begin{array}{c}\text { Vitek } 2 \\
\text { GP }\end{array}$ & $\begin{array}{c}\text { API } 20 \\
\text { Strep }\end{array}$ & $\begin{array}{l}\text { S. gallolyticus } \\
\text { subsp. } \\
\text { gallolyticus }\end{array}$ & $\begin{array}{l}\text { S. gallolyticus } \\
\text { subsp. } \\
\text { pasteurianus }\end{array}$ & $\begin{array}{l}\text { S. gallolyticus } \\
\text { subsp. } \\
\text { macedonicus }\end{array}$ & $\begin{array}{l}\text { S. infantarius } \\
\text { subsp. } \\
\text { infantarius }\end{array}$ & $\begin{array}{l}\text { S. infantarius } \\
\text { subsp. } \\
\text { coli }\end{array}$ \\
\hline \multicolumn{8}{|l|}{ Hydrolysis of: } \\
\hline Esculin & NT & + & + & + & - & v & + \\
\hline \multicolumn{8}{|l|}{ Production of: } \\
\hline$\beta$-Glucosidase & NT & + & + & + & - & v & + \\
\hline$\beta$-Glucuronidase & - & - & - & + & - & - & - \\
\hline$\alpha$-Galactosidase & + & - & + & $\mathrm{v}$ & $v$ & + & + \\
\hline$\beta$-Galactosidase & - & - & - & + & $\mathrm{v}$ & - & - \\
\hline \multicolumn{8}{|l|}{ Acidification of: } \\
\hline Starch & NT & + & + & - & + & + & v \\
\hline Glycogen & NT & + & + & - & - & + & - \\
\hline Inulin & NT & - & + & - & - & - & - \\
\hline Lactose & + & + & + & + & + & + & + \\
\hline Mannitol & + & + & + & - & - & - & - \\
\hline Methyl- $\beta$-D-glucopyranoside & + & NT & + & + & - & - & + \\
\hline Raffinose & + & + & + & v & - & + & - \\
\hline Trehalose & + & + & + & + & - & - & - \\
\hline
\end{tabular}

*The biochemical profiles of the subspecies of $S$. gallolyticus and $S$. infantarius were obtained from a report by Schlegel et al. [4]. Abbreviations: NT, not tested;,$+ \geq 80 \%$ of strains positive;,$- \leq 20 \%$ of strains positive; $v, 21-79 \%$ activity compared to the positive control reaction. 
identified as $S$. bovis.

\section{DISCUSSION}

The relationship between streptococcal endocarditis and colon cancer was first described in 1951 [6], and S. bovis was recognized as the pathogen specifically related to colon cancer in 1977 [1]. Although many other bacterial strains have been associated with gastrointestinal tumors, the association between $S$. bovis and colon cancer remains the strongest and best documented one. Several prospective studies have suggested that $S$. bovis infection could be seen as a first sign of colon cancer [7, 8]. Therefore, clinicians should not readily rule out colon cancer or other malignancies when they encounter patients with $S$. bovis infection. In this case, colon cancer can be detected by colonoscopy upon clinical suspicion of $S$. bovis infection, even without clinical findings suggesting colon cancer. The pathogenesis of the association between $S$. bovis infection and colon neoplasms has been widely investigated; however, the pathogenesis is still not clear. One hypothesis is that the $S$. bovis group is a normal inhabitant of the gastrointestinal tract and can readily enter the bloodstream upon mucosal disruption or vascular changes [9].

Since the early 1980s, genetic and biochemical diversities among $S$. bovis bacteria have been recognized. These diversities led to devising schemes to distinguish strains by biotype. S. bovis bacteria are said to be biotype I (or typical) if they ferment mannitol and produce glucan, and biotype II (or variant) if they cannot ferment mannitol or produce glucan. The $S$. bovis biotype II strains are further divided into types II/1 and II/2 according to the ability of the latter group to produce $\beta$-galactosidase and $\beta$-glucuronidase and ferment trehalose but not glycogen [10]. In the 1990s, 4 new species were described: Streptococcus gallolyticus, Streptococcus macedonicus, Streptococcus waius, and Streptococcus infantarius [11]. S. gallolyticus, originally isolated from koalas, is phenotypically similar to $S$. bovis biotype I [12], and reexamination of strains previously identified as $S$. bovis by either phenotypic or genetic methods revealed that many of the human strains associ- ated with endocarditis and animal (goat, pigeon, and cow) strains are identical to $S$. gallolyticus $[13,14]$. Meanwhile, molecular technologies, such as DNA-DNA hybridization or the amplification of selected targets, have been developed to complement and improve the identification of streptococci at the species level. Farrow et al. demonstrated that the S. bovis/Streptococcus equinus complex comprised 6 DNA groups [15]. Members of DNA group 2 have been reclassified, according to their genetic and biochemical characteristics, and renamed as $S$. gallolyticus subsp. gallolyticus, S. gallolyticus subsp. pasteurianus, or S. gallolyticus subsp. macedonicus [4]. Biochemical characteristics, such as gallate hydrolysis activity, are useful for the identification at the subspecies level. A recent study showed that the published phenotypic characteristics of $S$. gallolyticus and $S$. infantarius were equivocal and did not allow unambiguous phenotypic differentiation [5]. Beck et al. also showed that although the 16S rDNA gene sequences are very similar between subspecies, full-length 16S rDNA gene sequence analysis clearly assigned the subspecies in most cases because of the extremely low base pair variability within one species or subspecies. They showed that only 3 of 29 S. gallolyticus subsp. gallolyticus strains, 1 of $12 S$. gallolyticus subsp. pasteurianus strains, and 1 of $17 \mathrm{~S}$. infantariuss subsp. coli had single point mutations, whereas other strains showed identical 16s rDNA sequences within each subspecies [5]. Our isolates did not ferment inulin but fermented mannitol and raffinose. They also produced acid from starch, glycogen, methyl- $\beta-D-$ glucopyranoside, and trehalose, but did not produce $\beta$-glucuronidase and $\beta$-galactosidase (Table 1). On the basis of the biochemical characteristics and the 16S rDNA sequencing data, we could identify the infecting organism as $S$. gallolyticus subsp. gallolyticus with high confidence at the subspecies level.

Cases of $S$. bovis infection associated with underlying malignancies have been rarely reported in Korea. There are 2 previous reported cases of $S$. bovis endocarditis and associated colon cancer in Korea [16, 17]; however, they did not provide information about the microbiological characteristics or $S$. bovis biotypes. In a more recent singlecenter study, the association of $S$. bovis biotypes with the 
type of clinical infection and data on antimicrobial susceptibility was studied. In this study, none of the 13 patients with $S$. bovis bacteremia had gastrointestinal malignancies [18]. More studies on the prevalence, underlying diseases, and microbiological characteristics of $S$. gallolyticus subspecies in Korea are needed.

This case shows that accurate identification of $S$. gallolyticus subsp. gallolyticus endocarditis makes the detection of underlying cancer possible. Much attention should be given when identifying the causative organisms of streptococcal endocarditis, and investigation for underlying gastrointestinal malignancies should be performed when $S$. gallolyticus is identified. In addition, some clinicians may not be familiar with the new nomenclature system of $S$. bovis bacteria. This lack of awareness can lead to underdiagnosis of serious underlying conditions, including colon cancer; thus, it is important to provide clinicians with sufficient information about the nomenclature change when reporting study results. Moreover, although the use of the name $S$. bovis biotype I or II/2 instead of $S$. gallolyticus is common in clinical microbiology despite the clearly established new nomenclature, it is also important to update and use the proper taxonomic species in reporting, communication, or clinical studies to prevent further confusion in the future.

\section{REFERENCES}

1. Klein RS, Recco RA, Catalano MT, Edberg SC, Casey JI, Steigbigel $\mathrm{NH}$. Association of Streptococcus bovis with carcinoma of the colon. N Engl J Med 1977;297:800-2.

2. Ruoff KL, Miller SI, Garner CV, Ferraro MJ, Calderwood SB. Bacteremia with Streptococcus bovis and Streptococcus salivarius: clinical correlates of more accurate identification of isolates. J Clin Microbiol 1989;27:305-8.

3. Herrero IA, Rouse MS, Piper KE, Alyaseen SA, Steckelberg JM, Patel R. Reevaluation of Streptococcus bovis endocarditis cases from 1975 to 1985 by $16 S$ ribosomal DNA sequence analysis. J Clin Microbiol 2002;40:3848-50.

4. Schlegel L, Grimont F, Ageron E, Grimont PA, Bouvet A. Reappraisal of the taxonomy of the Streptococcus bovis/Streptococcus equinus complex and related species: description of Streptococcus gallolyticus subsp. gallolyticus subsp. nov., S. gallolyticus subsp. macedonicus subsp. nov. and S. gallolyticus subsp. pasteurianus subsp. nov. Int J Syst Evol Microbiol 2003;53:631-45.

5. Beck M, Frodl R, Funke G. Comprehensive study of strains previously designated Streptococcus bovis consecutively isolated from human blood cultures and emended description of Streptococcus gallolyticus and Streptococcus infantarius subsp. coli. J Clin Microbiol 2008;46:2966-72.

6. Mc CW and Mason JM 3rd. Enterococcal endocarditis associated with carcinoma of the sigmoid; report of a case. J Med Assoc State Ala 1951;21:162-6.

7. Klein RS, Catalano MT, Edberg SC, Casey JI, Steigbigel NH. Streptococcus bovis septicemia and carcinoma of the colon. Ann Intern Med 1979;91:560-2.

8. Wilson WR, Thompson RL, Wilkowske CJ, Washington JA 2nd, Giuliani ER, Geraci JE. Short-term therapy for streptococcal infective endocarditis. Combined intramuscular administration of penicillin and streptomycin. JAMA 1981;245:360-3.

9. Ellmerich S, Scholler M, Duranton B, Gosse F, Galluser M, Klein JP, et al. Promotion of intestinal carcinogenesis by Streptococcus bovis. Carcinogenesis 2000;21:753-6.

10. Coykendall AL. Classification and identification of the viridans streptococci. Clin Microbiol Rev 1989;2:315-28.

11. Poyart C, Quesne G, Trieu-Cuot P. Taxonomic dissection of the Streptococcus bovis group by analysis of manganese-dependent superoxide dismutase gene (sodA) sequences: reclassification of Streptococcus infantarius subsp. coli as Streptococcus lutetiensis sp. nov. and of Streptococcus bovis biotype 11.2 as Streptococcus pasteurianus sp. nov. Int J Syst Evol Microbiol 2002;52:1247-55.

12. Schlegel L, Grimont F, Collins MD, Regnault B, Grimont PA, Bouvet A. Streptococcus infantarius sp. nov., Streptococcus infantarius subsp. infantarius subsp. nov. and Streptococcus infantarius subsp. coli subsp. nov., isolated from humans and food. Int J Syst Evol Microbiol 2000; 50 Pt 4:1425-34.

13. Clarridge JE 3rd, Attorri SM, Zhang Q Bartell J. 16 S ribosomal DNA sequence analysis distinguishes biotypes of Streptococcus bovis: Streptococcus bovis Biotype II/2 is a separate genospecies and the predominant clinical isolate in adult males. J Clin Microbiol 2001;39:1549-52.

14. Devriese LA, Vandamme P, Pot B, Vanrobaeys M, Kersters K, Haesebrouck F. Differentiation between Streptococcus gallolyticus strains 
of human clinical and veterinary origins and Streptococcus bovis strains from the intestinal tracts of ruminants. J Clin Microbiol 1998;36: 3520-3.

15. Farrow JA, Kruze J, Phillips BA, Bramley AJ, Collins ME. Taxonomic studies on Streptococcus bovis and Streptococcus equinus: description of Streptococcus alactolyticus sp. nov. and Streptococcus saccharolyticus sp. nov. Syst Appl Microbiol 1984;5:467-82.

16. Kwack KK, So SC, Park HK, Lee DK, Kim JH, Lyu DY, et al. A case of subacute infective endocarditis with colon cancer caused by Streptococcus bovis. Korean J Med 2000;59:198-202.

17. Koh DW, Choi JH, Kim YS, Koo JS, Cho YJ, Park DK, et al. A case of two synchronous colon cancers accompanied by Streptococcus bovis endocarditis. Korean J Gastrointest Endosc 2001;23:503-6.

18. Uh Y, Kwon O, Yoon KJ, Hwang GY, Kim HY. Underlying diseases associated with Streptococcus bovis bacteremia and antimicrobial susceptibility of the organism. Korean J Clin Microbiol 2006;9:36-41. 